Published in final edited form as:

Eur Urol. 2017 June ; 71(6): 866-871. doi:10.1016/j.eururo.2016.12.026.

\title{
A Systematic Approach to Discussing Active Surveillance with Patients with Low-risk Prostate Cancer
}

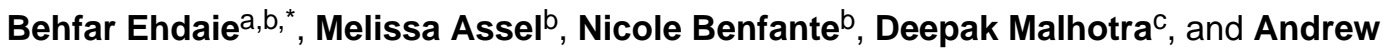 \\ Vickers $^{b}$ \\ aUrology Service, Department of Surgery, Memorial Sloan Kettering Cancer Center, New York, \\ NY, USA \\ bHealth Outcomes Research Group, Department of Epidemiology and Biostatistics, Memorial \\ Sloan Kettering Cancer Center, New York, NY, USA \\ 'Negotiation, Organizations, and Markets Unit, Harvard Business School, Boston, MA, USA
}

\section{Abstract}

Background-Physicians report difficulty convincing patients with prostate cancer about the merits of active surveillance (AS); as a result, a majority of patients unnecessarily choose to undergo radical treatment.

Objective-To develop and evaluate a systematic approach for physicians to counsel patients with low-risk prostate cancer to increase acceptance of AS.

Design, setting, and participants-A systematic counseling approach was developed and piloted in one clinic. Then five surgeons participated in a 1-h training session in which they learned about the approach. A total of 1003 patients with Gleason $3+3$ prostate cancer were included in the study. We compared AS rates for 761 patients who were counseled over a 24-mo

\footnotetext{
"Corresponding author. Urology Service, Department of Surgery, Memorial Sloan Kettering Cancer Center, 353 East 68th Street, New York, NY 10065, USA. Tel. +1 646 4224406. ehdaieb@ mskcc.org (B. Ehdaie).

Publisher's Disclaimer: This is a PDF file of an unedited manuscript that has been accepted for publication. As a service to our customers we are providing this early version of the manuscript. The manuscript will undergo copyediting, typesetting, and review of the resulting proof before it is published in its final citable form. Please note that during the production process errors may be discovered which could affect the content, and all legal disclaimers that apply to the journal pertain.

Author contributions: Behfar Ehdaie had full access to all the data in the study and takes responsibility for the integrity of the data and the accuracy of the data analysis.

Study concept and design: Ehdaie, Vickers, Malhotra.

Acquisition of data: Benfante, Assel, Ehdaie.

Analysis and interpretation of data: Ehdaie, Vickers, Malhotra, Assel.

Drafting of the manuscript: Ehdaie, Vickers, Malhotra, Benfante, Assel.

Critical revision of the manuscript for important intellectual content: Ehdaie, Vickers, Malhotra.

Statistical analysis: Assel, Benfante.

Obtaining funding. Ehdaie, Vickers.

Administrative, technical, or material support: Ehdaie.

Supervision: Vickers.

Other: None.

Financial disclosures: Behfar Ehdaie certifies that all conflicts of interest, including specific financial interests and relationships and affiliations relevant to the subject matter or materials discussed in the manuscript (eg, employment/affiliation, grants or funding, consultancies, honoraria, stock ownership or options, expert testimony, royalties, or patents filed, received, or pending), are the following: None.
} 
period before the training intervention with AS rates for 242 patients who were counseled over a 12-mo period afterwards, controlling for temporal trends and case mix.

Intervention-A systematic approach for communicating the merits of AS using appropriate framing techniques derived from principles studied by negotiation scholars.

Outcome measurements and statistical analysis-The rate of AS acceptance by patients for management of low-risk prostate cancer.

Results and limitations-In the pilot phase, 81 of 86 patients (93\%) accepted AS after counseling by the physician who developed the counseling approach. In the subsequent study, the cohort for the training intervention comprised 1003 consecutive patients, $80 \%$ of whom met the Epstein criteria for very low-risk disease. The proportion of patients who selected AS increased from $69 \%$ before the training intervention to $81 \%$ afterwards. After adjusting for time trends and case mix, the rate of AS after the intervention was $9.1 \%$ higher (95\% confidence interval $-0.4 \%$ to $19.4 \%$ ) than expected, a relative reduction of approximately $30 \%$ in the risk of unnecessary curative treatment.

Conclusions-A systematic approach to counseling can be taught to physicians in a 1-h lecture. We found evidence that even this minimal intervention can decrease overtreatment. Our novel approach offers a framework to help address cancer screening-related overtreatment that occurs across medicine.

Patient summary-In this study, we evaluated the impact of teaching physicians how to better communicate the benefits and risks of prostate cancer treatments on the willingness of patients to choose active surveillance. Decisions related to cancer are often guided by emotions and biases that lead most patients to seek radical treatment; however, we demonstrated that if discussions are framed differently, these biases can be overcome and more patients will choose active surveillance.

\section{Keywords}

Prostate cancer; Outcomes; Communication; Social psychology; Behavioral economics; Active surveillance

\section{Introduction}

The adoption of widespread prostate-specific antigen (PSA) screening has been accompanied by overdetection and subsequent overtreatment of select prostate cancers that are unlikely to lead to morbidity or mortality [1]. Active surveillance (AS) as a treatment modality attempts to reduce overtreatment of low-risk prostate cancer and involves careful, frequent monitoring, with subsequent curative treatment if evidence of cancer progression is found. Despite the desire to avoid potential morbidity associated with radical treatment (including erectile dysfunction and urinary or bowel incontinence) most men eligible for AS instead undergo curative treatment [2]. Fewer than $40 \%$ of all men with low-risk prostate cancer are currently managed with AS, and between $25 \%$ and $80 \%$ of patients undergo radical treatment for prostate cancer unnecessarily. Such overtreatment is commonly attributed to the misaligned incentives for physicians [3]. However, even those who support AS as a management approach report considerable difficulty in convincing patients of its merits [4]. Patients generally believe that cancer is inherently life-threatening, and so the 
perception that they would not be receiving curative treatment for their cancer causes significant anxiety [5]. Physicians receive very little, if any, training to effectively counsel patients about AS and may lack the communication skills necessary to address biases against a noncurative approach to management. As a result, patients rarely report discussing all the treatment options with physicians and often perceive AS as "doing nothing" [6]. These observations not only raise the possibility of achieving better patient outcomes but also suggest potential to reduce health care costs. One study estimated that if $50 \%$ of patients recently diagnosed with low-risk prostate cancer were to choose AS, the health care savings would surpass $\$ 1$ billion in the USA annually [7].

Scholars in the field of negotiation have studied various methods for achieving better agreements between individuals who have different perspectives or seemingly divergent interests [8,9]. Furthermore, considerable research among social psychologists and behavioral economists reveals that decision-making is impacted by how options are "framed", such as whether consequences are described in terms of lower costs or greater benefits. When physicians provide options and education about low-risk prostate cancer, they are inevitably making several (potentially unconscious) choices on how these are framed, including the order in which treatment options are communicated and whether consequences are described in terms of gains (eg, "survival rates are higher") or losses (eg, "death rates are lower") $[10,11]$. These framing choices, whether made deliberately or not, can impact patient choice.

Other well-studied principles in behavioral science that can impact patient decisions include "social proof" (how the choices made by similar individuals in similar situations influence our own choices) and "reference point effects" that shift the context within which a decisionmaker evaluates an option [10,12]. For example, educating patients about the relatively long latent natural history of prostate cancer before describing the follow-up schedule for AS can overcome the perception that the schedule is not aggressive enough, or that the cancer can metastasize in the time between serial examinations. Studies have demonstrated that these framing effects impact medical decision-making [13,14].

An important unmet need is to help physicians engage patients about all treatment options for low-risk prostate cancer in a way that is ethically responsible, and takes into account biases that might encourage immediate intervention even in situations for which there is little if any mortality benefit but potentially significant costs in terms of morbidity.

Our systematic approach to counseling men first evokes, as advised by negotiation scholars, all of the patient's own interests - that is, their reasons for considering the various treatment options. For example, a patient who is interested only in reducing mortality risk is less likely to consider noncurative treatments than one who acknowledges an interest in reduced mortality and morbidity. Once a patient has articulated his interests, appropriate framing principles can help the physician to effectively communicate how AS can be a viable treatment option for the perceived life-threatening malignancy. This represents a novel approach with the potential to help patients with low-risk prostate cancer avoid unnecessary radical treatment. 
The approach was adopted by one of our urologists (B.E.) in his clinic, and led to a seemingly large increase in the number of patients accepting AS. Accordingly, we sought to determine whether this experience could be generalized to other physicians. As a first step, we decided to test a minimal intervention in which negotiation theory (which focuses on the importance of identifying and addressing underlying interests) and social psychology principles (to effectively frame options) were taught in a single 1-h lecture. Here, we report the rate of AS acceptance by patients who were counseled by physicians before and after the minimal teaching intervention.

\section{Patients and methods}

\subsection{Development of a systematic approach to counseling prostate cancer patients}

We conducted a review of qualitative studies exploring the perceptions of AS among patients with prostate cancer and their families to identify factors influencing treatment selection and beliefs about the efficacy and side effects of immediate treatment options [6,15-19]. In analyzing these factors, we outlined a conceptual framework describing barriers to AS acceptance centered on six themes: (1) lack of information about AS; (2) attitudes and beliefs that cancer is life-threatening and should be treated; (3) the perception that AS is best suited for older patients who are not candidates for surgery or radiation treatment; (4) belief that immediate aggressive treatment would lead to better survival; (5) anxiety and fear that cancer will spread without detection; and (6) persuasion against AS by family members. In collaboration with a scholar in the field of negotiation who has experience using appropriate framing techniques derived from social psychology and behavioral economics (D.M.), we formalized a systematic approach to counseling patients regarding all treatment options for low-risk prostate cancer. The approach targets the barriers to AS acceptance described above and addresses the tendency of patients to heavily favor immediate treatment on the basis of perceptions and beliefs that are sometimes inaccurate. After the approach was piloted in one clinic (B.E.), the intervention was revised in accordance with feedback from the scholar in negotiation principles (D.M.) after he directly observed the physician interaction with patients (Supplementary material).

\subsection{Teaching physicians the systematic approach to counseling patients}

We recruited five physicians from the urology service at Memorial Sloan Kettering Cancer Center (MSKCC) who specialize in prostate cancer to participate in a training program teaching the systematic approach to counsel patients with prostate cancer. The systematic approach was introduced through a 60-min didactic lecture and reinforced with case scenarios. After the training, each physician received a pocket-sized booklet that reiterated the framing principles and described how they could be applied to prostate cancer decisionmaking.

\subsection{Patient cohort}

We collected data for all patients with clinical and pathologic features consistent with lowrisk prostate cancer who had an initial consultation visit from August 2012 to August 2015. These features included Gleason grade $\leq 3+3$ prostate cancer, clinical stage $\leq \Gamma 2 \mathrm{~A}$, and PSA $\leq 10 \mathrm{ng} / \mathrm{ml}$. Patients who chose AS were recommended to undergo a confirmatory 
prostate biopsy performed at our institution to determine eligibility for AS. All pathology slides were reviewed and confirmed at MSKCC by pathologists specialized in genitourinary oncology.

\subsection{Evaluating the effectiveness of the systematic approach to counseling patients}

We conducted an observational study measuring the rate of AS for patients with low-risk prostate cancer before and after the training intervention. The intervention date was August 8,2014 . One concern was that negotiation would increase consultation times. A surrogate for consultation time is clinic room time. The clinic room time includes any time during which the patient was speaking with the physician, nurse, or fellow, and includes any additional waiting time while in the examination room. Although it is not a specific measure of the actual consultation time, if an increase in consultation time occurred, it would be observed in the clinic room time. As a secondary outcome, we measured the change in mean initial clinic room time for counseling men with newly diagnosed low-risk prostate cancer before and after the intervention. We used routinely collected administrative data to gather information regarding each patient's treatment choice and clinic room time. The treatment plan was determined by combining data in the electronic health record including: a physician's recommendation documented in the initial consultation note; a phone call documented by nurses to follow-up with patients $1 \mathrm{wk}$ after the initial consultation; documented definitive therapy before confirmatory prostate biopsy at MSKCC or an outside institution; or an AS visit and/or confirmatory prostate biopsy before treatment. In addition, we reviewed all patients' charts 6 mo after the initial consultation to verify the treatment decision.

\subsection{Statistical analysis}

We first calculated the rate of AS acceptance in the pilot clinic. We then compared the rate at which patients of the five physicians exposed to the teaching intervention selected AS during the 24-mo time period before the intervention with the rate during the $12 \mathrm{mo}$ after the intervention. The analysis followed a previously described statistical methodology that controls for trends occurring in the time period before the intervention [20]. Using the data from the 24-mo period before the intervention, we developed a statistical model predicting the probability of a patient selecting AS on the basis of age, comorbidity, ethnicity, tumor classification (Epstein low-risk criteria vs other), and PSA level before diagnostic biopsy, and to control for secular trends from the date of each patient's initial clinic visit. AS rates observed after the intervention were compared to those predicted by the model, with the standard error for the difference between observed and predicted values calculated via bootstrapping. We repeated this analysis to compare clinic room time before and after the intervention. All analyses were conducted using Stata 13 (Stata Corp., College Station, TX, USA).

\section{Results}

The systematic approach was implemented in the pilot clinic on January 1, 2014. Between January 1, 2014 and August 8, 2014, the rate of AS acceptance in this clinic was 93\% (39 out of 42 patients). This rate remained steady at $94 \%$ ( 81 out of 86 patients) in the following 
12 mo. To evaluate the impact of the teaching intervention, we collected data for 1003 consecutive patients with Gleason $\leq 3+3$ prostate cancer, clinical stage $\leq \mathrm{T} 2 \mathrm{a}$, and PSA $\leq 0$ $\mathrm{ng} / \mathrm{ml}$ who were counseled by one of the five participating physicians. Of these patients, $80 \%$ met the Epstein criteria for very low-risk disease. The patient characteristics are presented in Table 1. We found no significant differences in patient characteristics for initial consultations before and after the intervention. The percentage of patients who selected AS before the training intervention increased from $69 \%$ (95\% confidence interval [CI] 66-73\%) to $81 \%$ (95\% CI 76-86\%) following the intervention (Fisher's exact test, $p=0.001$ ). The effect was slightly attenuated after adjusting for temporal trends in AS and for case mix. The percentage of patients enrolled in AS was $9.1 \%$ (95\% CI -0.4\% to 9.4\%) higher than expected (Fig. 1). Given a $31 \%$ rate of treatment at baseline, a $9 \%$ absolute risk difference is equivalent to a relative reduction in the risk of overtreatment of close to $30 \%$.

The mean clinic room time for patients at initial consultation was 79 and $77 \mathrm{~min}$ before and after the intervention, respectively. After adjusting for the time trend and case mix, we did not find evidence to suggest a difference in the mean clinic room time, and therefore found no evidence that consultation time was longer after the intervention (Fig. 2).

\section{Discussion}

Our study shows that implementation of a systematic counseling approach by a committed surgeon can result in extremely high adoption (93\%) of AS by patients. We further demonstrated that surgeons with no prior experience or educational background in negotiation or behavioral decision-making can be taught the basics of this approach in a single 1-h training session. The systematic counseling approach led to an approximate $30 \%$ reduction in overtreatment of patients with low-risk prostate cancer. Importantly, we did not see any evidence that incorporation of the systematic counseling approach increased clinic consultation times. The systematic approach to counseling patients was taught to the five participating physicians by another physician (B.E.) who had no experience in negotiation or behavioral science before working closely with a scholar in negotiation (D.M.). Therefore, a train-the-trainer model is a promising method for efficient dissemination of these tools to physicians across larger health systems.

Urologic surgeons dedicate upwards of a decade in training to become experts in radical prostatectomy for the treatment of prostate cancer. This complex procedure follows a highly structured, carefully studied sequence of steps that require significant repetition to achieve proficiency; however, the majority of contemporary patients are diagnosed with low-risk prostate cancer that does not require immediate treatment and can be carefully managed with AS. Studies show that physicians are keen to adopt standardization for other interventions, such as checklists to decrease variability, and thus error, when performing serial tasks. Despite this, physicians do not follow a set of principles when counseling patients regarding alternative treatment options, especially in the current context of prostate cancer in which immediate intervention is heavily favored [21,22]. In this study, we demonstrated that a systematic approach to counseling patients with low-risk prostate cancer using appropriate framing techniques derived from the social sciences can be taught to physicians and can lead 
to improved acceptance of AS. As a result, many men are spared unnecessary treatment and consequent urinary, sexual, and bowel dysfunction.

The management of localized prostate cancer is largely preference-based, and comparative effectiveness studies have yet to demonstrate an advantage of a single treatment modality, highlighting the complexities of treatment decision-making for patients. Furthermore, studies have found biases or heuristics at work in patient decision-making that heavily favors intervention $[6,15,17,23,24]$. Biases or heuristics heavily favor intervention for patients with low-risk prostate cancer and raise ethical questions as to whether patients are making rational or optimal decisions. A proposal to overcome these barriers is to engage in ethically responsible choice architecture in which physicians incorporate well-studied principles from behavioral decision-making that include appropriate framing techniques to encourage patients to not ignore the harms of immediate treatment or the benefits of AS [25].

We adapted these principles to the needs of patients with low-risk prostate cancer and developed a systematic approach to counseling patients. Importantly, we did not provide physicians with a script of what to say. Therefore, we did not record counseling sessions between patients and physicians. Our goal was to teach physicians communication techniques derived from scholars in negotiation to incorporate as they determined to be most effective according to their own personal counseling style. Similarly, our focus was on changing patient behavior, not patient beliefs; therefore, we did not measure patient anxiety or decisional conflict. We believe that this represents a strength in our approach and will facilitate broader dissemination of these communication tools among physicians.

After physicians were taught the systematic approach to counseling patients, the rate of AS increased from $69 \%$ to $81 \%$. Following adjustment for time trends and case mix, the increase in AS acceptance did not reach the conventional definition of statistical significance. Therefore, we cannot exclude the possibility that the $30 \%$ decrease in overtreatment was due to other factors. This includes a Hawthorne effect associated with physicians knowingly being observed regarding AS rates, or increasing acceptance of AS among patients during the study period. However, we believe that the short proposed study period mitigates the possibility of a large-scale change in AS perceptions among patients and physicians. Importantly, the Prostate Cancer Intervention versus Observation Trial (PIVOT), a landmark study demonstrating that observation for prostate cancer is a viable option, was published a year before the beginning of our study period. All of the physicians participating in our study are specialized in prostate cancer management and supported AS before the study period. Indeed, the baseline rate of AS adoption, close to 70\%, is far higher than comparable estimates from the literature. Current rates of AS in low-risk cancer in community settings range from $35 \%$ to $45 \%$ [2]. Our center is a specialized tertiary hospital in which patients are most often already diagnosed with prostate cancer and are seeking treatment on the basis of discussions with primary care providers or other urologists. Therefore, we believe that our intervention may be even more effective in clinical practices in which physicians manage patients from diagnosis through to intervention, as these elements in communication can be used before prostate biopsy and diagnosis of cancer. Furthermore, the importance of incorporating an approach to counsel patients is highlighted by the most current American Society of Clinical Oncology guideline, which states that AS 
should be the preferred option for all men with low-risk disease, regardless of tumor volume [26].

We observed a measurable effect size despite the limited training session in which physicians with no background in negotiation or framing techniques were exposed to a 1-h didactic lecture, and with no follow-up sessions to observe, modify, and improve the physician-patient counseling approach. In this context, we believe that expanding the training and incorporating validated communication skills training can improve the effect size [27]. Despite the improvement in AS acceptance after the intervention, we did not see evidence of an increase in consultation time. Although mean clinic room time may not be an exact measure of consultation time, we were interested to identify large increases in consultation time using the novel elements in communication, and we found no evidence of a large increase in consultation time on the basis of changes in mean clinic room time. Furthermore, our primary outcome was to assess acceptance of AS by patients newly diagnosed with prostate cancer, so evaluation of long-term adherence is beyond the scope of this study. Therefore, we believe that the reasons for non-adherence to AS can be evaluated in future research proposals, and modifications to the communication strategy can be implemented for visits after the initial consultation. A limitation of a single-institution study is generalization of the intervention effect across varied geographic locations, ethnicities, and patient socioeconomic categories. Despite the differences between patients seen in a single tertiary center and the general population of patients, the aim of the communication intervention is not to change physicians' beliefs but to help physicians who support AS to more effectively communicate the merits of AS to eligible patients. We plan to conduct follow-up studies to observe, modify, improve, and test the approach further in a multiinstitutional trial.

\section{Conclusions}

A systematic approach to counseling men with prostate cancer using appropriate framing techniques adapted from behavioral social science principles can be taught to physicians and effectively incorporated in the clinic to reduce the burden of overtreatment without increasing consultation times. This novel approach was developed to help address heuristics and biases in patients who favor immediate intervention for management of low-risk prostate cancer and to encourage men with low-risk prostate cancer to consider AS. We believe that the results of this study have relevance beyond prostate cancer. Our approach provides a framework to advance the physician-patient relationship and may help in alleviating the overdiagnosis and overtreatment that occur across medicine.

\section{Supplementary Material}

Refer to Web version on PubMed Central for supplementary material.

\section{Acknowledgments}

Funding/Support and role of the sponsor: This work was supported in part by funds from David H. Koch provided through the Prostate Cancer Foundation, the Sidney Kimmel Center for Prostate and Urologic Cancers, P50CA92629 SPORE (Principal Investigator: Howard I. Scher) and P30 CA008748 (Principal Investigator Craig B. 
Thompson). The sponsors played a role in the design and conduct of the study, management and analysis of the data, and review and approval of the manuscript.

\section{References}

1. Etzioni R, Penson DF, Legler JM, et al. Overdiagnosis due to prostate-specific antigen screening: lessons from U.S. prostate cancer incidence trends. J Natl Cancer Inst. 2002; 94:981-90. [PubMed: 12096083]

2. Cooperberg MR, Carroll PR. Trends in management for patients with localized prostate cancer, 1990-2013. JAMA. 2015; 314:80-2. [PubMed: 26151271]

3. Jacobs BL, Zhang Y, Skolarus TA, Hollenbeck BK. Growth of high-cost intensity-modulated radiotherapy for prostate cancer raises concerns about overuse. Health Affairs. 2012; 31:750-9. [PubMed: 22492892]

4. Kim SP, Gross CP, Nguyen PL, et al. Perceptions of active surveillance and treatment recommendations for low-risk prostate cancer: results from a national survey of radiation oncologists and urologists. Med Care. 2014; 52:579-85. [PubMed: 24926704]

5. van den Bergh RC, Essink-Bot ML, Roobol MJ, et al. Anxiety and distress during active surveillance for early prostate cancer. Cancer. 2009; 115:3868-78. [PubMed: 19637245]

6. Xu J, Dailey RK, Eggly S, Neale AV, Schwartz KL. Men's perspectives on selecting their prostate cancer treatment. J Natl Med Assoc. 2011; 103:468-78. [PubMed: 21830629]

7. Hayes JH, Ollendorf DA, Pearson SD, et al. Observation versus initial treatment for men with localized, low-risk prostate cancer: a cost-effectiveness analysis. Ann Intern Med. 2013; 158:85360. [PubMed: 23778902]

8. Malhotra D, Bazerman MH. Investigative negotiation. Harv Bus Rev. 2007; 85:72-6.

9. Fisher, R., Ury, W. Getting to yes: negotiating agreement without giving. 2. New York, NY: Penguin Books; 1991. p. 15-81.

10. Tversky A, Kahneman D. The framing of decisions and psychology of choice. Science. 1981; 211:453-8. [PubMed: 7455683]

11. Kahneman D, Tversky A. Prospect theory: an analysis of decision under risk. Econometrica. 1979; 47:263-91.

12. Cialdini RB, Goldstein NJ. Social influence: compliance and conformity. Annu Rev Psychol. 2004; 55:591-621. [PubMed: 14744228]

13. McNeil BJ, Pauker SG, Sox HC Jr, Tversky A. On the elicitation of preferences for alternative therapies. N Engl J Med. 1982; 306:1259-62. [PubMed: 7070445]

14. Kalichman SC, Coley B. Context framing to enhance HIV-antibody-testing messages targeted to African American women. Health Psychol. 1995; 14:247-54. [PubMed: 7641666]

15. Xu J, Neale AV, Dailey RK, Eggly S, Schwartz KL. Patient perspective on watchful waiting/active surveillance for localized prostate cancer. J Am Board Fam Med. 2012; 25:763-70. [PubMed: 23136314]

16. Mishra MV, Bennett M, Vincent A, et al. Identifying barriers to patient acceptance of active surveillance: content analysis of online patient communications. PLoS One. 2013; 8:e68563. [PubMed: 24039699]

17. Penson DF. Factors influencing patients' acceptance and adherence to active surveillance. J Natl Cancer Inst Monogr. 2012; 45:207-12.

18. Pickles T, Ruether JD, Weir L, Carlson L, Jakulj F, SCRN Communication Team. Psychosocial barriers to active surveillance for the management of early prostate cancer and a strategy for increased acceptance. BJU Int. 2007; 100:544-51. [PubMed: 17532857]

19. Srirangam SJ, Pearson E, Grose C, Brown SC, Collins GN, O'Reilly PH. Partner's influence on patient preference for treatment in early prostate cancer. BJU Int. 2003; 92:365-9. [PubMed: 12930420]

20. Vickers AJ, Cronin AM, Masterson TA, Eastham JA. How do you tell whether a change in surgical technique leads to a change in outcome? J Urol. 2010; 183:1510-4. [PubMed: 20172569] 
21. Holmes-Rovner M, Montgomery JS, Rovner DR, et al. Informed decision making: assessment of the quality of physician communication about prostate cancer diagnosis and treatment. Med Decision Making. 2015; 35:999-1009.

22. Zikmund-Fisher BJ, Couper MP, Singer E, et al. Deficits and variations in patients' experience with making 9 common medical decisions: the DECISIONS survey. Med Decision Making. 2010; $30(5$ Suppl):85S-95S.

23. Fagerlin A, Zikmund-Fisher BJ, Ubel PA. Cure me even if it kills me: preferences for invasive cancer treatment. Med Decision Making. 2005; 25:614-9.

24. Denberg TD, Melhado TV, Steiner JF. Patient treatment preferences in localized prostate carcinoma: the influence of emotion, misconception, and anecdote. Cancer. 2006; 107:620-30. [PubMed: 16802287]

25. Blumenthal-Barby JS, Lee D, Volk RJ. Toward ethically responsible choice architecture in prostate cancer treatment decision-making. CA Cancer J Clin. 2015; 65:257-60. [PubMed: 25998159]

26. Chen RC, Rumble RB, Loblaw DA, et al. Active surveillance for the management of localized prostate cancer (Cancer Care Ontario Guideline): American Society of Clinical Oncology Clinical Practice Guideline endorsement. J Clin Oncol. 2016; 34:2182-90. [PubMed: 26884580]

27. Kissane DW, Bylund CL, Banerjee SC, et al. Communication skills training for oncology professionals. J Clin Oncol. 2012; 30:1242-7. [PubMed: 22412145] 
A systematic counseling approach may help to address heuristics and biases among patients who favor immediate intervention for their cancers and increase acceptance of active surveillance for prostate cancer; thereby preventing overtreatment of low-risk prostate cancers. 


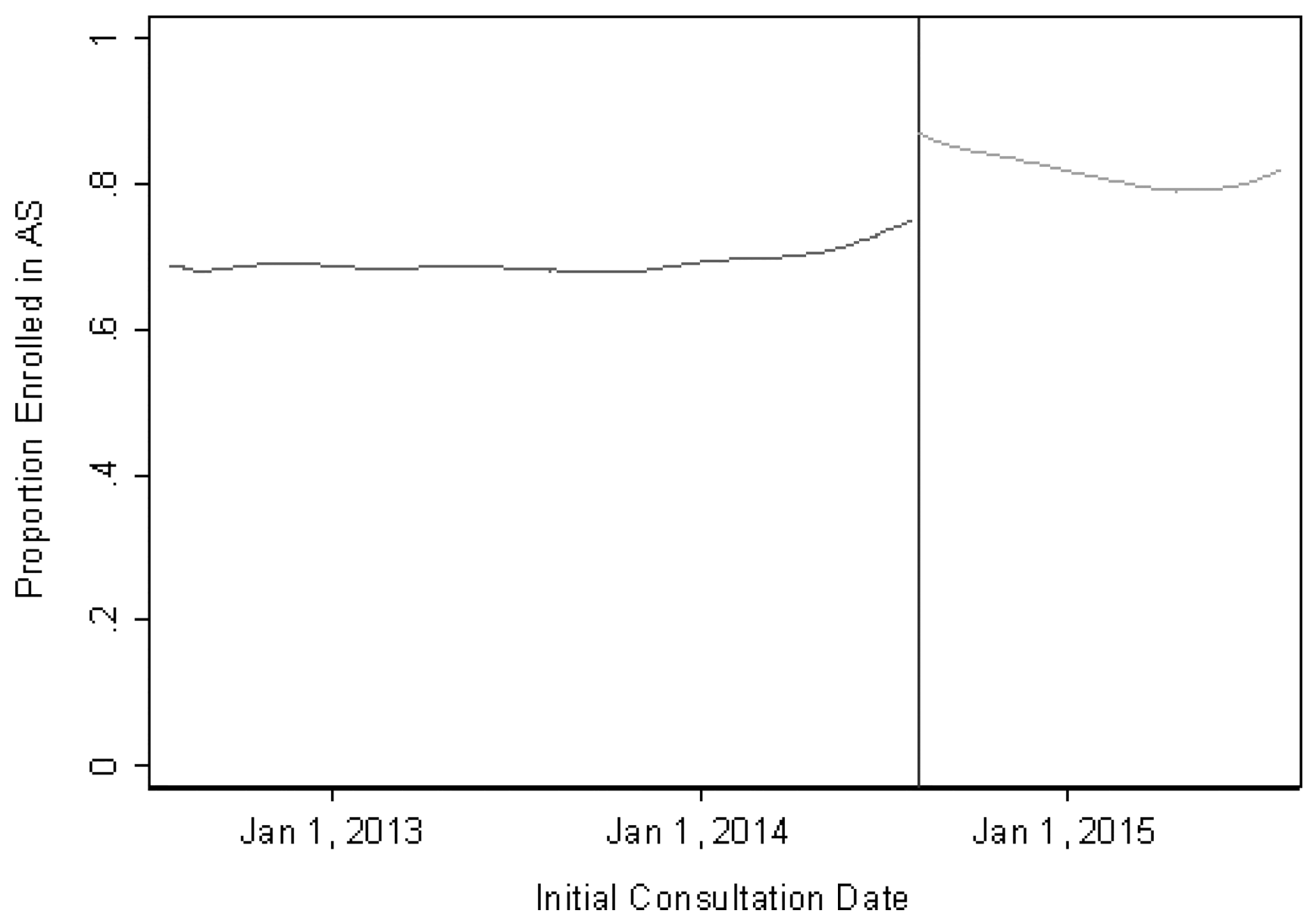

Fig. 1.

Proportion of patients who enrolled in active surveillance (AS) by initial consultation date. The vertical line represents the date of the intervention. 


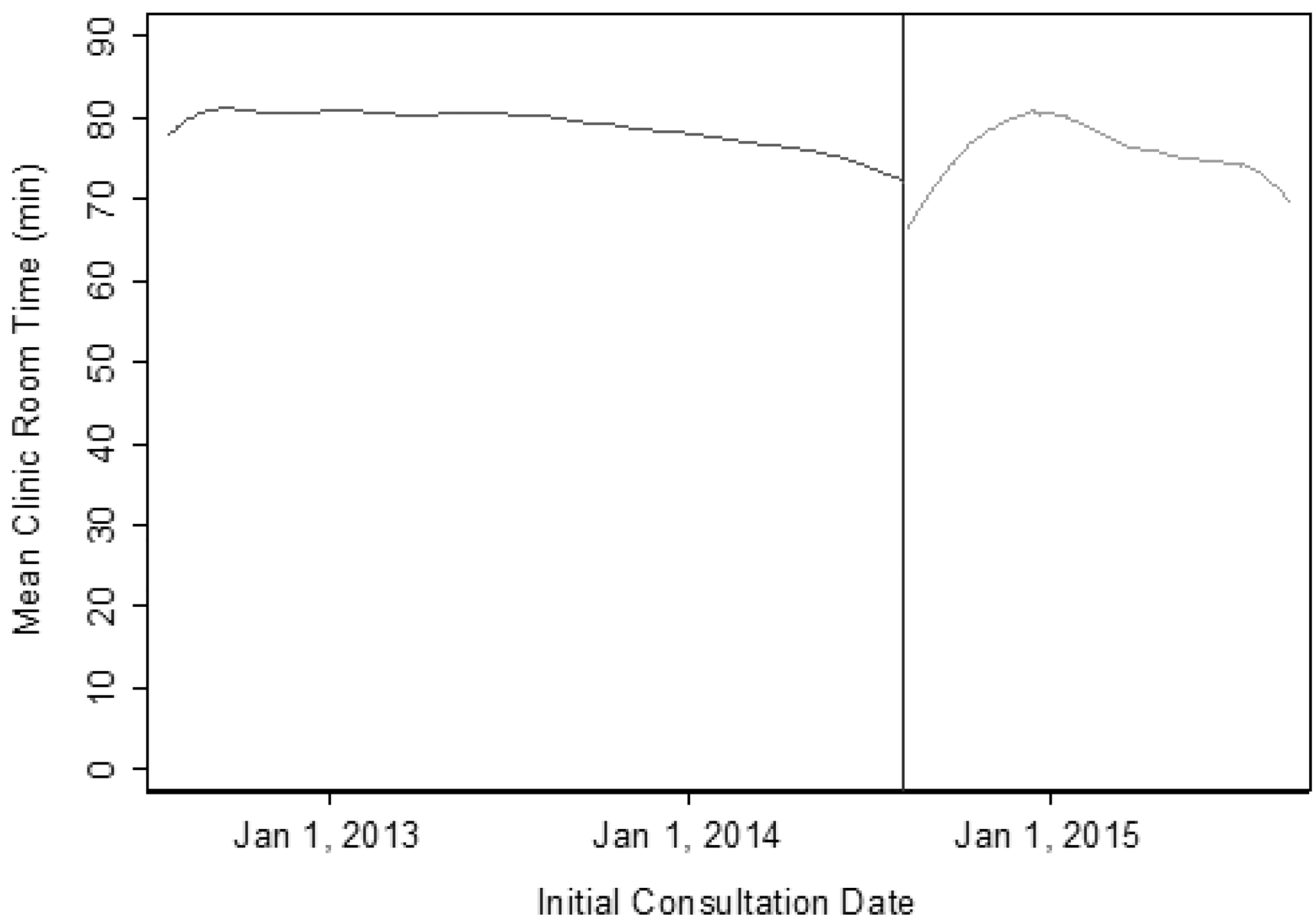

Fig. 2.

Mean initial clinic room time by initial consultation date. The vertical line represents the date of the intervention. 


\section{Table 1}

Patient characteristics by initial consultation group

\begin{tabular}{llll}
\hline Characteristic & \multicolumn{1}{l}{ Patient cohort } & p value \\
\cline { 2 - 3 } & $\begin{array}{l}\text { Pre-intervention } \\
(\boldsymbol{n = 7 6 1 ; 7 6 \% )})\end{array}$ & $\begin{array}{l}\text { Post-intervention } \\
(\boldsymbol{n}=\mathbf{2 4 2} \text { 24\% })\end{array}$ & \\
\hline Age (yr) & $60(55-66)$ & $60(55-66)$ & 0.9 \\
Charlson comorbidity index & & $211(87)$ & 0.091 \\
0 & $628(83)$ & $31(13)$ & \\
$\geq 1$ & $133(17)$ & $20(8.3)$ & 0.3 \\
Race & & $204(84)$ & \\
White & $86(11)$ & $18(7.4)$ & \\
Other & $650(85)$ & & \\
Unknown & $25(3.3)$ & $190(79)$ & 0.6 \\
Tumor classification & & $52(21)$ & \\
Very low risk & $611(80)$ & $4.7(3.7-5.9)$ & 0.5 \\
\hline Low risk & $150(20)$ &
\end{tabular}

$\mathrm{PSA}=$ prostate-specific antigen.

Data are presented as median (interquartile range) for continuous variables and $\mathrm{n}(\%)$ for categorical variables. To determine $p$ values, the Wilcoxon rank sum test was applied for continuous variables and Fisher's exact test for categorical variables. 